\title{
Segmentation and Organization of Lecture Video Based On Visual Contents
}

\author{
Nilesh J. Uke and Ravindra C. Thool
}

\begin{abstract}
In multimedia-based learning systems, there is need for segmenting and organizing lecture videos into topic and subtopics. Fundamental problem in any lecture video is to give semantic query and effectively retrieve relevant contents form long video. Effective search capability for the students can be provided if proper browsing facility is provided. Automatic segmentation is highly desired because of the high cost of manual segmentation. In this paper, we propose a general system for segmenting and organizing method for educational videos that is based on a knowledge base of existing recorded lectures. Our approach concentrates on the slides instead of a presenter from the projected electronic slides. The proposed method aims in using the video streams of the lecture delivered captured from camera and get the slides converted into images after applying slide segmentation. These independent images have heading and subheadings which will be used for organizing the lecture video.

The organizing and segmenting videos of the technical videos according to slide changes not only structure the content of video according to topics, but also facilitate the synchronization of video, audio and electronic slides for effective indexing, browsing and retrieval at later stage.
\end{abstract}

Index Terms-Browsing, multimedia, video segmentation.

\section{INTRODUCTION}

Videotaping of lecture video is more common in CBT (Computer Based Training) and e-Learning. Many researchers have shown that multimedia instruction can enhance students' problem-solving and understanding skills [1]. The content of most lecture videos cover more than one topic or sub-topic. In order to facilitate student learning and minimize learning time, lecture videos usually are segmented into smaller topics for browsing [2]. Generally lectures take at least one to two hour and will produce a large video file. Consequently, it becomes difficult for learners to access the lecture content efficiently.

Now days in professional colleges like IIT's, NIT's and other Engineering colleges are also making video of their lecture videos. The main objective of NPTEL program is to enhance the quality of engineering education in the country by developing curriculum based video and web courses. This is being carried out by seven IITs and IISc Bangalore as a collaborative project [3]. In the first phase of the project, supplementary content for 129 web courses in engineering/science and humanities have been developed.

Manuscript received October 9, 2011; revised March 02, 2012

N. J. Uke is with Department of Information Technology, Sinhgad College of Engineering, Pune, India (e-mail address: njuke.scoe@sinhgad.edu).

R. C. Thool is with Department of Information Technology, SGGS College of Engineering and Technology, Nanded, India.
But students find it difficult to access the relevant topic in the long video recorded earlier.

Segmenting these videos into small clips and organizing them into browsing structures such as table of contents and index will improve the problem of unnecessary searching for exact contents. Therefore, the objective of this study is to develop effective and efficient automated approaches to segment lecture videos into topics/subtopics. This video segmentation and browsing structures will support knowledge searching and e-Learning.

\section{RELATED WORK}

Usage of lecture video for educational purposes is a topic that has been addressed at least since the 1970s. Chan use the lecture notes along with Optical Character Recognition (OCR) techniques to synchronize the video with the text. A hierarchical index is formed by analyzing the original lecture text to extract different levels of headings. An underlying assumption is made that the slides are organized as a hierarchy of topics, which is not always the case. Many slides may have titles which are in no way related to the previous slide.

Proposed a system using a human intermediary (the teacher) [4], [5] as an interpreter to manually index the video. Hwang et al. propose a hyper video editor tool to allow the instructor to mark various portions of the class video and create the corresponding hyperlinks and multimedia features to facilitate the students' access to these pre-recorded sequences through a web browser. This scheme also requires a human intermediary and thus is not generalized.

The recently developed COVA system offers browsing and querying in a lecture database; however, it constructs the lecture index using a digital text book and neglects other sources of information such as audio or PowerPoint slides.

Microsoft supported on-line training lectures with more than 9000 online viewers in the year of 1999 alone. These elearning systems enhance learning experiences and augment teachers' work in and out of traditional classrooms. Working professionals as well embrace e-learning programs due to their convenience and flexibility. However, due to unstructured and liner features of videos, the essential instructional content of most e-learning systems, the presentation videos, has not been fully exploited [6].

There are several approaches where work inclines towards KM (Knowledge management) instead of organization of lecture video, $\mathrm{KM}$ as viewed from the perspective of a student taking a course for the first time, or exploring a course to review a particular concept. The BMRC lecture browser [7] provides a web based platform for the viewing of lecture videos with synchronized slides 
and a table of contents for the visualization of the lecture content. The IBM lecture browser emphasizes the use of automatic analysis of the video and the generation of transcripts from speech to support content based access. Whereas other lecture browsers were designed to facilitate the access and playback of lecture content, the emphasis of lecture explorer is on the interactive visualization of lecture content and the use of pedagogical structures to facilitate task based exploration of the learning material.

\section{OVERVIEW OF THE SYSTEM}

\section{A. A Shot (Slide)Boundary Detection}

Lecture videos are different from normal videos and have very little shot/boundary change [8]. Therefore images cues are not available for such type of application. But audio of lecture video can be very rich for using process of segmentation into topic and subtopic. In this system audio part is omitted. Most of the technical lecture videos start with a title slide, then an outline/overview slide, which is followed by a number of content slides. These content slides normally have block diagrams, examples, case studies and other text related to the contents. The outline/overview slide of a presentation summarizes major topics that will be covered in content slides. It is also seen that the any introduction of a new topic in the content slides generally uses terms that are the same as or very similar to what occur in the outline/overview slides. This helps us in extracting headings and subheadings as an index term in the browsing structure.

In our system we are interested in conducting initial video recording for the lectures conducted using power point presentation instead of black board writing. Since today teachers in professional colleges are using multimedia presentation for conducting lectures. We need initial video shot boundary detection algorithm which will be based on changes in slides. Much short boundary detection approaches have been proposed by various researchers. Here we use slide boundary detection module which will helps in detecting a new topic or subtopic from the lecture video. We conduct certain type of heuristic approach in finding out the important slide. Change in headings, block diagrams, case studies, theorems and important phrase shall be considered as a slide change.

\section{B. Slide Level Segmentation (Adding Index Point)}

System needs to add an index point at the important slide change detected. This slide level segmentation process divides the continuous video stream into set of video frames each of which models one individual frame/image of the presentation. Given a video stream vp and a set of $n$ slides, we can compute a set of slide video segment as

$$
\mathrm{vS}_{\text {slide }}=\left\{\mathrm{vS}_{0}, \mathrm{vS}_{1}, \ldots \mathrm{vS}_{\mathrm{v}}\right\}
$$

Such that slide image of each video segment $\mathrm{vS}_{\mathrm{i}}$ do not change for $0<=\mathrm{i}<=\mathrm{m}$. Actually this segmented video sequence $\mathrm{vS}_{\mathrm{i}}$ display the same slide in the presentation. We get many similar slides in the presentation lasting for few seconds to minutes. We need to take a single index point for such type of slide level segmentation shown in the Fig. 1.

\section{Topic and Subtopic Organization}

Normally a power point presentation will have heading and subheading, which will helps us to get topics and subtopics for organization. A typical presentation, especially a lecture in a class, starts with a title slide, then an outline/overview slide, which is followed by a number of content slides [8]. The outline slide of a presentation summarizes major topics that are to be covered.

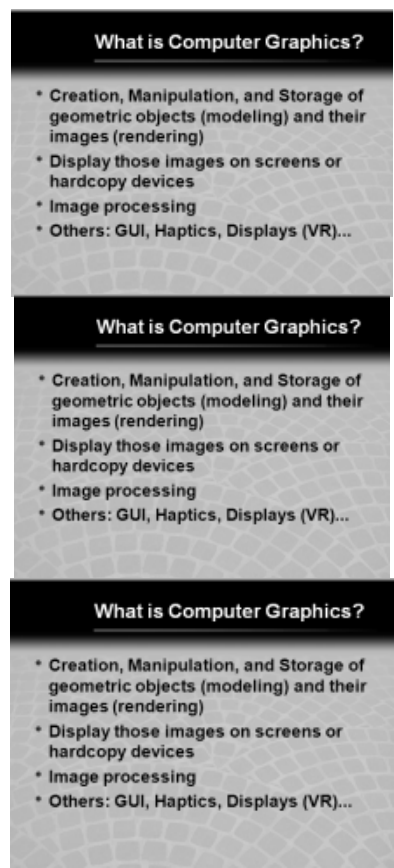

Fig. 1. Slide level segmentation showing same slide.

We may also have some slide with the same heading nut different contents. It is necessary to give a separate index point for such slides images, as shown in the image of the Fig. 2.

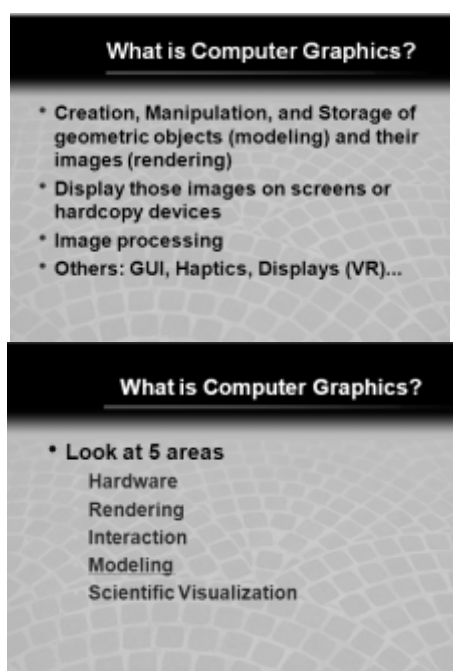

Fig. 2. Slide level segmentation showing different slide.

\section{Block Diagram of Proposed System}

Initially the lecture video is captured using a stationary digital camera. We assume that camera motion is very less. Power point slides are screened through projector and displayed on screen. General block diagram is shown in Fig 3.

In our study, we observed that most of the presentations 
specially class lecture, starts with the title slide, then the outline or overview slide and then followed by current slides. Following Fig 2 and Fig 3 shows slides captured and converted to image having heading and subheading in the slide, which will be used for organization the entire lecture video.

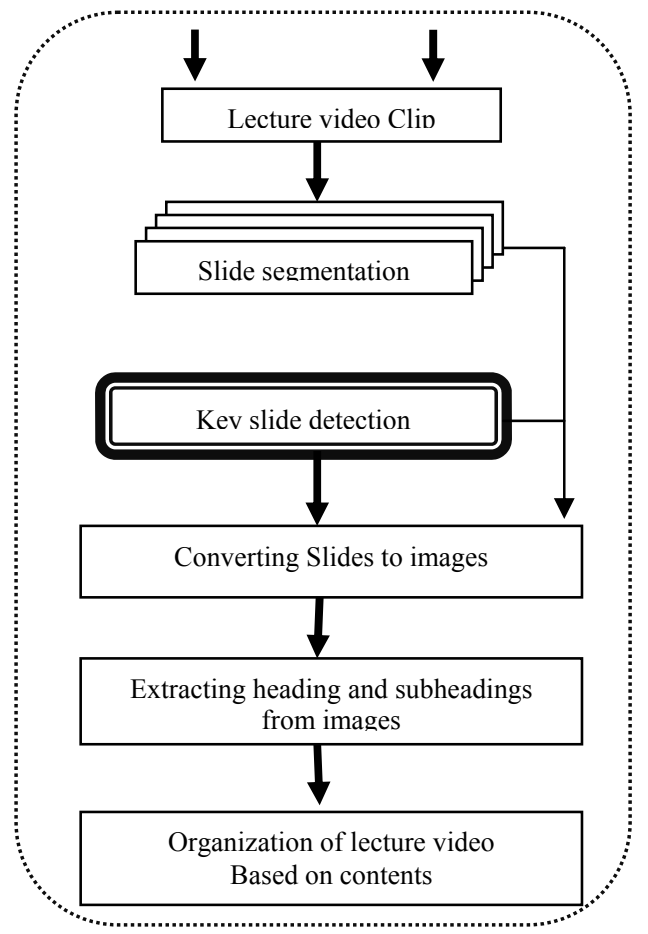

Fig. 3. Block diagram of proposed system.

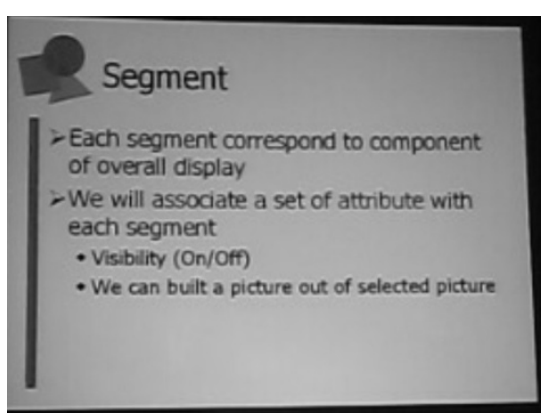

Fig. 4. Slide converted to Image.

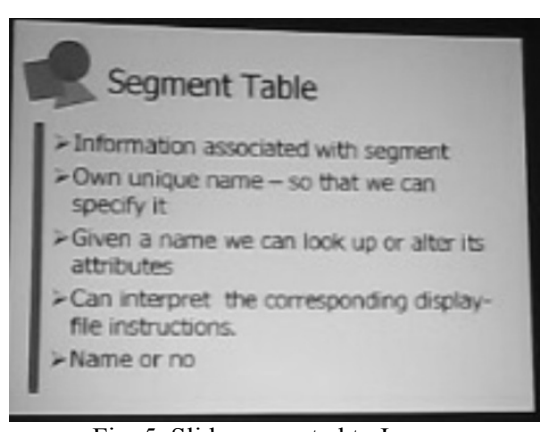

Fig. 5. Slide converted to Image.

\section{CONCLUSION}

Video plays an important role in today's education. This growth of video data demands efficient and easy access mechanisms. This paper describes the early stage of the development of segmentation and organization of lecture video based on visual contents. To extract the headings and subheading data from videos and video segments and to organize them in a way that facilitates lecture video access, we propose a simple vision method based system of extracting important image frames with the help of index points. We envision that there will be more extensive research conducted to effectively segment, annotate, and access these data, thus making them fully benefit the advance of society.

Learning techniques are not restricted to time or place, but new learning methods will help students in gaining the insight of the actual thought.

\section{ACKNOWLEDGEMENTS}

This work was partially funded by Board of College and University Department (BCUD), University of Pune, Pune. The authors also wish to acknowledge the unknown referee, for the useful suggestions.

\section{REFERENCES}

[1] W. Zhang, Chapter 8, "Multimedia Technology, Education and Learning", Technological Innovations in Literacy and Social Studies Education [online], 1995. Available: http://tiger.core.missouri.edu

[2] M. R. Halvorsen, "Content-based lecture video indexing," Master's thesis ,2007.

[3] NPTEL (National Programme on Technology Enhanced Learning), http://nptel.iitm.ac.in

[4] A. Bibiloni and R. Galli, "Content Based Retrieval Video System for Educational Purposes," In Proceedings of Eurographics Workshop on Multimedia on the Net, EGMM 1996, 2003.

[5] Y.-C. Liao and C.-H. Huang, "Automatic Video Segmentation and Story-Based Authoring in E-Learning," Journal of Software, vol. 4, no. 2, April 2009

[6] A. Mittal, P. V. Krishnan, and E. Altman, "Content Classification and Context-Based Retrieval System for E-Learning," Educational Technology \& Society, vol. 9, no. 1, pp. 349-358.

[7] L. A. Rowe, J. M. Gonzlez, BMRC lecture browser demo. http://bmrc.berkeley.edu/frame/projects/lb/index.htm

[8] A. Dong and H. Li, "Ontology-driven annotation and access of Presentation video data," Journal of Theoretical and Applied Information Technology, vol. 4, no. 9, 2008. 